\title{
Sensitivity of the spherical gravitational wave detector MiniGRAIL operating at 5 K
}

\author{
L. Gottardi, ${ }^{*}$ A. de Waard, O. Usenko, and G. Frossati \\ LION, Institute of Physics, Kamerlingh Onnes Laboratorium, Leiden University, Leiden, The Netherlands \\ M. Podt ${ }^{\dagger}$ and J. Flokstra \\ Low Temperature Division, Faculty of Science and Technology, Twente University, Enschede, The Netherlands \\ M. Bassan, V. Fafone, Y. Minenkov, and A. Rocchi \\ Dipartimento di Fisica, Università Tor Vergata and INFN Roma Tor Vergata, Roma, Italy \\ (Received 1 May 2007; revised manuscript received 2 October 2007; published 21 November 2007)
}

\begin{abstract}
We present the performances and the strain sensitivity of the first spherical gravitational wave detector equipped with a capacitive transducer and readout by a low noise two-stage SQUID amplifier and operated at a temperature of $5 \mathrm{~K}$. We characterized the detector performance in terms of thermal and electrical noise in the system output signal. We measured a peak strain sensitivity of $1.5 \times 10^{-20} \mathrm{~Hz}^{-1 / 2}$ at $2942.9 \mathrm{~Hz}$. A strain sensitivity of better than $5 \times 10^{-20} \mathrm{~Hz}^{-1 / 2}$ has been obtained over a bandwidth of $30 \mathrm{~Hz}$. We expect an improvement of more than 1 order of magnitude when the detector will operate at $50 \mathrm{mK}$. Our results represent the first step towards the development of an ultracryogenic omnidirectional detector sensitive to gravitational radiation in the $3 \mathrm{kHz}$ range.
\end{abstract}

DOI: 10.1103/PhysRevD.76.102005

PACS numbers: 04.80.Nn, 02.60.Pn, 95.55.Ym

\section{INTRODUCTION}

The direct observation of gravitational waves (GWs) is one of the most challenging tasks for experimental physics. After the first detection is claimed a new branch of astronomical observation will begin and gravitational wave observatories will become more and more common facilities. A spherical detector is an outstanding instrument for an astronomical observatory due to its feature of omnidirectionality and polarization sensitivity [1-5]. The first ultracryogenic spherical gravitational wave detectors [6,7] are currently completing their engineering phase and will soon be operational with an expected sensitivity better than $10^{-21} \mathrm{~Hz}^{-1 / 2}$ at $3 \mathrm{kHz}$. We report the results of the first sensitive measurement of the spherical gravitational wave detector MiniGRAIL. The detector readout consists of capacitive resonant transducers coupled by means of superconducting transformers to linear amplifiers based on superconducting quantum interference devices (SQUIDs). The two-stage SQUID described in this work is one of the most sensitive amplifiers ever used on a gravitational wave antenna. It consists of a sensor dcSQUID amplified by a double relaxation oscillation squid (DROS). We obtained a coupled additive energy resolution of $700 \hbar$ at $5 \mathrm{~K}$ in agreement with the expected values calculated from the device parameters using the standard SQUID model [8]. The spherical antenna described in this paper is the first example of a multimodal resonant detector where the five quadrupolar modes of the sphere are read

\footnotetext{
${ }^{*}$ Current address: SRON, Netherlands Institute for Space Research, Utrecht, the Netherlands.

l.gottardi@sron.nl

Current address: Thales Nederland B.V., Hengelo, The Netherlands.
}

out by three resonant transducers. In this paper we discuss the noise contribution and the signal response of one readout channel and estimate the detector sensitivity when the detector will operate at $50 \mathrm{mK}$ with a complete readout. This paper is organized as follows. In Sec. II we described the experimental apparatus and, in particular, the readout system. In Sec. III we present and discuss the experimental results. In Sec. III A and III B we analyze the electrical system, the noise spectra and equivalent temperature of the resulting coupled oscillators. Finally in Sec. III C we describe the calibration procedure and estimate the detector strain sensitivity. The work presented here is strongly linked to the recently published review on the spherical detectors [9]. We refer the reader to this article for a more detailed theoretical description of the concepts and the analysis reported here.

\section{SYSTEM OVERVIEW}

\section{A. Sphere and mechanical transducers}

MiniGRAIL is a spherical gravitational wave (GW) antenna currently under development [6]. The antenna is a massive sphere in $\mathrm{CuAl}$, has a diameter of $68 \mathrm{~cm}$, a physical mass $m_{s} \sim 1.3$ ton and the GW sensitive spheroidal quadrupole modes have frequencies around $2980 \mathrm{~Hz}$ at $T=4.2 \mathrm{~K}$. The alloy $\mathrm{CuAl} 6 \%$ has been chosen because of the high quality factor ( $Q \sim 10^{7}$ at low temperature), high sound velocity $\left(V_{S} \simeq 4100 \mathrm{~m} / \mathrm{s}\right)$ and a sufficient thermal conductivity, which allows the cooling of a 1.3 ton antenna to a temperature below $100 \mathrm{mK}$ [10]. The ultimate goal is to operate MiniGRAIL at a thermodynamic temperature of $20 \mathrm{mK}$, equipped with six transducers coupled to nearly quantum limited double-stage SQUID amplifiers [11-13]. The sphere is suspended from its center with a gold-plated 
copper rod $20 \mathrm{~mm}$ in diameter that hangs from the last mass of the mechanical vibration isolation system. This system consists of the following (from the inside outward): (a) seven low-pass filtering stages (mass-spring) suspended with stainless steel cables from (b) three absorbers, each consisting of a stack of rubber and aluminum plates. A detailed description of the detector mechanics and cryogenics can be found in [14].

We used capacitive transducers to read out the spheroidal modes. They consist of a closed membrane with a load mass in the center. The electrode is made of a thin $\mathrm{CuAl}$ plate placed in front of the resonating mass. To obtain a small gap between resonator and electrode, we proceeded as follows. The resonator and the electrode are lapped and polished to get a smooth flat surface. Further a clean Kapton foil of a thickness equal to the desired gap is placed between the electrode and the mass. Finally a small amount of glue is added between the electrode and the support springs. A load is applied on top of the electrode in order to make a compact assembly. After the glue has cured, the Kapton foil is removed. This technique was shown to be reliable and reproducible. Gaps of the order of $20 \mu \mathrm{m}$ could be obtained and voltage bias as large as $500 \mathrm{~V}$ could be applied without discharging.

In the experiment described here the sphere was equipped with the three resonators located at position 1 , 2 and 5 of the truncated icosahedral (TI) arrangement shown in Fig. 1 of Ref. [9]. Two transducers, named transducer 1 and transducer 2, located at the TI positions 1 and 5 , were coupled, respectively, to the two-stage SQUID and to a single stage commercial quantum design (QD) SQUID [15]. The third resonator, transducer 3, at position 2, was coupled to a room temperature field-effect transistor (FET) amplifier and has been used for diagnostic and calibration purposes.

In Table I we summarize the features of the three transducers. Each resonator is tuned mainly to a different spherical mode, has a resonant mass $m_{t}$ of about $200 \mathrm{~g}$ and a mechanical quality factor $Q$ of about $10^{4}$ at $300 \mathrm{~K}$.

TABLE I. Properties of the three $\mathrm{CuAl} 6 \%$ closed membrane transducers. The transducers resonance frequencies have been estimated from the tuning procedure at room temperature as described in the text.

\begin{tabular}{lccc}
\hline \hline \multicolumn{4}{l}{ Closed membrane transducers } \\
& Transducer 1 & Transducer 2 & Transducer 3 \\
\hline$m_{t}[\mathrm{Kg}]$ & 0.205 & 0.153 & 0.150 \\
$C_{t}[\mathrm{nF}]$ & $1.17 \pm 0.01$ & $0.70 \pm 0.05$ & $1.20 \pm 0.05$ \\
$\mathrm{Gap} d_{0}[\mu \mathrm{m}]$ & $20 \pm 2$ & $35 \pm 4$ & $25 \pm 2$ \\
$f_{\text {res }}[\mathrm{Hz}]$ & $2863 \pm 5$ & $2850 \pm 5$ & $2878 \pm 5$ \\
$Q$ at $300 \mathrm{~K}$ & $1.0 \times 10^{4}$ & $1.1 \times 10^{4}$ & $1.0 \times 10^{4}$ \\
$Q$ at $77 \mathrm{~K}$ & $3 \times 10^{4}$ & $4.8 \times 10^{4}$ & $2.0 \times 10^{4}$ \\
\hline \hline
\end{tabular}

\section{B. The readout system}

To achieve omnidirectionality and the best sensitivity and bandwidth in a spherical detector, a configuration of at least 5 transducers is necessary. We will not give here a compelling demonstration of the powerful properties of a spherical GW detector.

The aim of the work presented here is to demonstrate, for the first time, the readout of a multimodal spherical resonant antenna at a sensitivity limited by the thermal properties of the detector using one single transducer based on a two-stage SQUID amplifier.

For this reason we will limit our analysis to the performance of transducer 1 coupled to a two-stage SQUID system based on a DROS [16,17], and, as sensor SQUID, a QD dcSQUID with input inductance $L_{i}=1.6 \mu \mathrm{H}$, SQUID inductance $L_{\mathrm{SQ}}=80 \mathrm{pH}, M_{i, \mathrm{SQ}} \simeq 10 \mathrm{nH}$ and expected flux noise at $4.2 \mathrm{~K}$ of $\sqrt{S_{\Phi}}=1.2 \mu \Phi_{0} / \sqrt{\mathrm{Hz}}$ [18]. The second readout channel, equipped with a single commercial QD SQUID, transducer 2, was added to the system as a backup and its performance will not be discussed any further. The mechanical contribution of its resonator is, however, significant in the output response of transducer 1 and the calibrator (transducer 3) and it is of course included in the analysis reported below.

The scheme of readout circuit of transducer 1 is shown in Fig. 1. The equations of motion of the electromechanical system are fully described in [9] and we will not derive them here. The transducer displacement, $x_{t}$, modulates the capacitor $C_{t}$ which is biased at a constant voltage $V_{b}$. The electric field across the transducer gap is given by $E=$ $V_{b} / d_{0}$, where $d_{0}$ is the gap between the modulating mass and the fixed electrode. The ac signal current $I_{p}$ is coupled to the SQUID input circuit through a superconducting transformer with $L_{p}$ and $L_{s}$ the inductances of the primary and secondary coils, respectively. In a standard configuration, like, for example, when a dc-SQUID is used as amplifier, $L_{s}$ is directly connected to the SQUID input coil $L_{i}$ evaporated on top of the dc-SQUID washer.

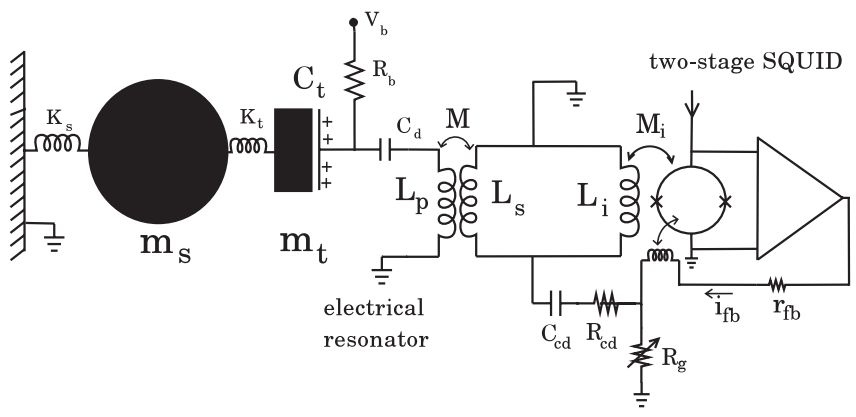

FIG. 1. Electromechanical scheme of a spherical antenna with mechanical resonator and capacitive transducer coupled to a SQUID through a superconducting matching transformer. A detailed scheme of the two-stage SQUID system can be found in [22]. 
The impedance matching between the transducer capacitance and the SQUID input coil is achieved by using the high-Q superconducting transformer. In our case, the electrical resonance of the transformer is not tuned to the mechanical modes, so the impedance matching is not optimal. The transformer coils were made of $\mathrm{Nb}$ wires and enclosed into a double superconducting shield. The bias circuit and the decoupling capacitor have been housed into a separate compartment of the superconducting shielding box. The measured transformer primary and secondary coil inductances are, respectively, $L_{p}=(0.3595 \pm$ $0.005) \mathrm{H}$ and $L_{s}=(2.1 \pm 0.2) \mu \mathrm{H}$. The mutual inductance between the coils was $M=(3.55 \pm 0.05) \times$ $10^{-4} \mathrm{H}$ and the coupling factor $\alpha=M / \sqrt{L_{p}\left(L_{s}+L_{i}\right)} \simeq$ 0.31 .

In the transducer bias line we use a decoupling Teflon commercial capacitor with capacitance $C_{d}=220 \mathrm{nF}$ and several bias resistors for a total resistance of $R_{b}=13 \mathrm{G} \Omega$ at $4.2 \mathrm{~K}$. The final intrinsic electrical quality factor, after connecting the decoupling capacitor $C_{d}$ and the bias resistor $R_{b}$, is equal to $Q=(1.8 \pm 0.1) \times 10^{5}$ at $4.2 \mathrm{~K}$. The transducer was assembled with a gap of about $(20 \pm$ 2) $\mu \mathrm{m}$, and a capacitance $C_{t}=(1.2 \pm 0.05) \mathrm{nF}$ measured at room temperature. This has been done in order to keep the electrical mode separated from the mechanical ones. The electrical mode resonance, resulting from the combination of the transducer capacitance and the reduced inductance $\quad L_{r}=L_{p}\left(1-M^{2} / L_{p}\left(L_{s}+L_{i}\right)=(0.325 \pm\right.$ $0.005) \mathrm{H}$, has a frequency of $f_{e l}=1 / 2 \pi \sqrt{L_{r} C_{t}}=$ $(8188.9 \pm 0.5) \mathrm{Hz}$ measured on the antenna at $5 \mathrm{~K}$. From the electrical mode frequency we estimate a total transducer capacitance of $C_{t}=(1.16 \pm 0.01) \mathrm{nF}$, which includes the effect of stray capacitances in parallel $\left(C_{\text {stray }} \sim 20 \mathrm{pF}\right)$ and the decoupling capacitance in series $\left(C_{d}=220 \mathrm{nF}\right)$.

Two-stage SQUID systems are developed in order to reduce the noise of dc-SQUID amplifiers, which are normally limited by the room temperature electronics [16,1921]. When used in the transducer chain for resonant gravitational waves detectors, they can improve the detection sensitivity by orders of magnitude [13]. The system described here differs from other two-stage SQUIDs used in GW experiments since it uses a DROS as an amplifier SQUID [17]. A DROS has a large flux-to-voltage transfer function which allows direct readout of the signal. Direct readout simplifies multichannel readout as needed in spherical gravitational wave detectors.

The two-stage SQUID system we developed is based on a configuration reported in [16,22]. A standard QD dcSQUID chip was chosen as sensor SQUID because of a larger input inductance with respect to the dc-SQUID described in [16].

The total additive flux noise of the two-stage SQUID, measured in a test dewar at $4.2 \mathrm{~K}$ was $\sqrt{S_{\Phi}^{-}}=(1.60 \pm$ $0.02) \mu \Phi_{0} / \sqrt{\mathrm{Hz}}$ with input coil open and $\sqrt{S_{\Phi}}=(1.10 \pm$
0.02) $\mu \Phi_{0} / \sqrt{\mathrm{Hz}}$ with input coil superconductively shorted. This corresponds, respectively, to an intrinsic uncoupled energy resolution of $\epsilon=S_{\Phi} / 2 L_{s q}=(650 \pm 15) \hbar$ and $\epsilon=$ $(320 \pm 15) \hbar$. This is in agreement with the expected values calculated from the SQUID parameters using the standard model [8]. In order to avoid instability in the SQUIDresonator system we implemented a capacitive cold damping network in the feedback line. Damping network has been first investigated by Stevenson [23], using a phaseshifted inductive feedback, and by Vinante [13], who made use of a capacitive network. The two-stage SQUID coupled to a high quality factor electrical resonator showed the same performances [18]. We estimate at $T=4.2 \mathrm{~K}$ a SQUID noise temperature $T_{N}=(100 \pm 30) \mu \mathrm{K}$ and a noise number $N=k_{B} T_{N} / \hbar \omega=730 \pm 100$. The additive coupled energy resolution was $650 \hbar$ and $320 \hbar$ respectively at $T=4.2$ and $2.1 \mathrm{~K}$.

\section{The calibrator}

To calibrate the mechanical vibration of the detector modes we used a piezoelectric transducer (PZT) and the transducer coupled to the FET amplifier transducer 3 that from now on we call calibrator. We performed the following calibration steps. First, we measure the electromechanical impedance of the calibrator by measuring with two-lock-in amplifiers the amplitude and the phase of the voltage and current across it. Then we excite each mode at resonance using the auxiliary PZT at a signal level well above the calibrator FET amplifier noise and, even more so, above the SQUID and transducer 1 noise. Finally we measure simultaneously the signal response of both the calibrator and the two-stage SQUID connected to transducer 1.

The electrical impedance $Z$ of the calibrator, for each resonant mode of frequency $\omega_{m}$ and quality factor $Q_{m}$, can be easily derived by solving the equations of motion of a capacitor, biased with an electric field $E_{\text {cal }}$, coupled to a mechanical resonating mass $m_{m}$. One finds

$$
Z(\omega)=\frac{1}{i \omega C_{\mathrm{cal}}}\left(1-\frac{C_{c a l} E_{\mathrm{cal}}^{2}}{m_{m}} \frac{1}{\omega_{m}^{2}-\omega^{2}+\frac{i \omega \omega_{m}}{Q_{m}}}\right) .
$$

The real part is used to estimate the energy of the mode and can be derived as follows

$$
\begin{aligned}
\operatorname{Re}[Z(\omega)] & =\frac{\omega_{m} E_{\mathrm{cal}}^{2}}{m_{m} Q_{m}} \frac{1}{\left(\omega_{m}^{2}-\omega^{2}\right)^{2}+\frac{\omega^{2} \omega_{m}^{2}}{Q_{m}^{2}}} \\
& =\frac{A_{m}}{\left(\omega_{m}^{2}-\omega^{2}\right)^{2}+\frac{\omega^{2} \omega_{m}^{2}}{Q_{m}^{2}}} .
\end{aligned}
$$

We notice that the real part of the impedance does not depend on the calibrator capacitance $C_{\text {cal }}$. This makes the calibration method described here particularly interesting because it is free from systematic errors, at least to the purpose of estimating the temperature of the mode. 
We remark here that in [9] a different and more direct way to calibrate the spherical detector is described. It is based on the use of a calibration coil weakly coupled to the matching transformer of the readout transducer as employed in [24] and a nonresonant capacitive transducer. A similar method is also described in [25]. The details of such a calibration procedure were not yet fully developed by the time of performing the experiment described here. For this reason another procedure was adopted here, which is less accurate due to the fact that the transducer itself cannot be calibrated directly. Moreover because only one excitation PZT was available, the strain sensitivity could only be derived for a particular incoming direction, which was almost optimal according to our detector simulations. We estimated an accuracy in the calibration of about $20 \%$. This value can be strongly improved in the future introducing minor changes in the setup as described in [9].

\section{EXPERIMENTAL RESULTS}

\section{A. Electrical system and noise spectrum}

A real sphere has five nondegenerate principal quadrupolar modes which are sensitive to GW. When mechanical resonators tuned to the quadrupolar modes are mounted on the sphere surface a splitting of the resonances occurs as described in detail in [26,27]. When $N$ transducers are added, $5+N$ main resonances are expected.

The resonators, completed with the electrodes, were attached to the sphere, one at a time. At room temperature, we measured for each transducer the frequency of the coupled mode. Fine tuning was performed by etching the transducer membrane and/or machining the resonator mass until the splitting of the modes was approximately the one expected for the transducer having a resonance frequency in the region of the quadrupolar modes. By solving numerically the equation of motion [26,27], it is possible to estimate the uncoupled resonator frequency by comparing the experimentally measured frequencies to the calculated ones and modifying the uncoupled resonator frequency in the calculation until a fair agreement with the experimental data is obtained.

The best agreement with the experimental data was found when the uncoupled resonant frequency of each transducer was set equal to $f_{1}=(2863 \pm 3) \mathrm{Hz}, f_{2}=$ $(2850 \pm 3) \mathrm{Hz}$ and $f_{3}=(2878 \pm 3) \mathrm{Hz}$. The error was estimated empirically by looking at how large the deviation between experimental and calculated frequencies was when varying the transducer uncoupled frequency.

In Fig. 2 we show the frequency measurements of the coupled modes when all three resonator were mounted on the sphere. The solid lines are the measured values while the dashed lines are the calculated values obtained by using a best fit for the uncoupled frequency of the resonators. We recall that these uncoupled frequencies cannot be measured independently because they depend too strongly on the fastening conditions. The calculated and measured mode

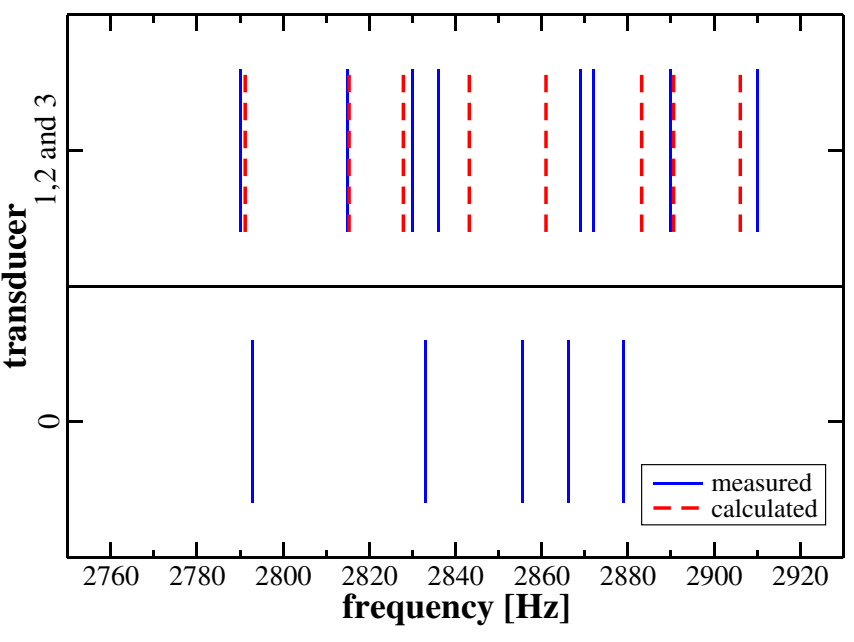

FIG. 2 (color online). Frequency measurement at room temperature of the coupled modes for transducers 1, 2 and 3 placed, respectively, on positions 1,5 and 2 compared with the bare sphere modes. The solid lines are the measured values and the dashed line are the calculated ones. The best values for the uncoupled transducers resonant frequencies are $f_{1}=(2863 \pm$ 3) $\mathrm{Hz}, f_{2}=(2850 \pm 3) \mathrm{Hz}$ and $f_{3}=(2878 \pm 3) \mathrm{Hz}$.

frequencies were fairly consistent with each other. This means that the coupled system is reasonably well described by the equation of motion derived in [9].

The disagreement between the calculated and measured frequency may be due to the departure from the geometry of a perfect sphere assumed in the equations of motion. For instance, the 6 pockets machined for hosting the transducers and the suspension hole were not considered.

A finite element analysis of the mechanical model could give a more accurate estimate of the frequencies.

The measurements discussed further on were performed at thermodynamic temperature $T=5.2 \mathrm{~K}$. Figure 3 shows the output of the two-stage SQUID when the transducer was biased at $10^{5} \mathrm{~V} / \mathrm{m}$. All the expected 8 modes of the system are visible in the spectrum.

The additive noise level of the two-stage SQUID coupled to the transducer mounted on the sphere, was comparable with the one measured with the SQUID with open input. When operating without the cold damping network, the minimum wideband flux noise observed with the SQUID was of $\sim 2.7 \mu \Phi_{0} / \sqrt{\mathrm{Hz}}$. When the cold damping was active, we measured an additive wideband flux noise of $(1.67 \pm 0.03) \mu \Phi_{0} / \sqrt{\mathrm{Hz}}$. It corresponds to an additive coupled energy resolution of $(730 \pm 100) \hbar$.

To establish which modes were strongly coupled to the transducer with the two-stage SQUID system, we used the fact that in a capacitive transducer the bias electric field introduces a negative spring constant, which causes the resonant frequency of the transducer resonator to shift down. In the simplest case, this shifted frequency is related to the transducers electromechanical coupling coefficient $\beta$ by $\beta=-\frac{\omega_{t}^{2}-\omega_{0}^{2}}{\omega_{t}^{2}}[28] . \beta$ is defined as the ratio between 


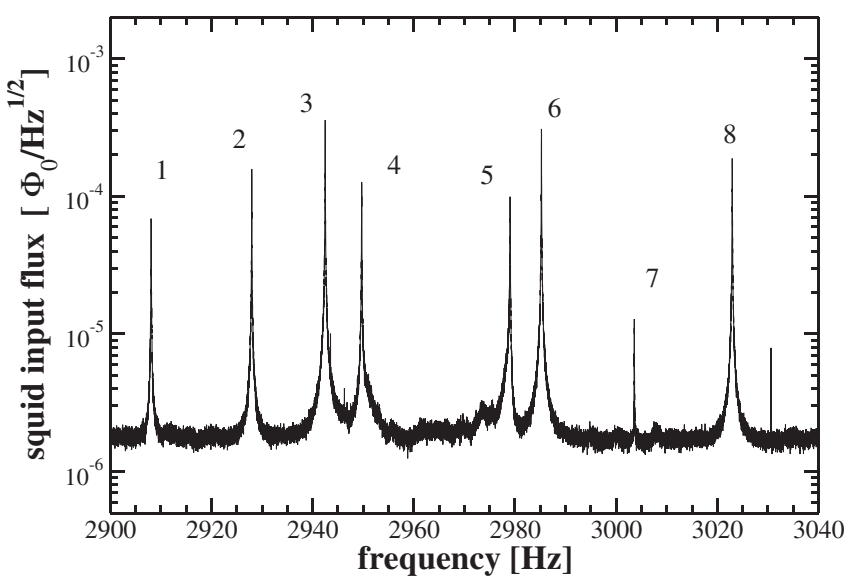

FIG. 3. Flux spectral density measured at the SQUID output with transducer bias at $200 \mathrm{~V}$. All the expected 8 modes of the system are visible in the spectrum.

the mechanical and the electrical energy, and describes the conversion efficiency of mechanical motion into electrical signal. It can be written as follows [29]: $\beta=\frac{1}{d_{0}^{2}} \frac{C_{t} t^{2}}{\omega^{2} m_{e f f}}$ where the effective mass $m_{\text {eff }}$ includes possible geometrical factors [30] as well as electrical parameters from the coupled circuit. The expected relation between the resonance frequency $f_{t}$ and the dc bias voltage $V_{b}$ is given by $f_{t}^{2}=f_{0}^{2}+\left(C_{t} / 4 \pi^{2} m_{e f f} d_{0}^{2}\right) V_{b}^{2}$ as observed in Fig. 4.

By measuring the frequency for different dc voltages applied to the transducer one gets an estimation of the electromechanical coupling and of the effective mass $m_{\text {eff }}$ if the transducer capacitance and the gap are known. The frequency measurements can be done very accurately by using a lock-in amplifier and monitoring the output phase $\phi$ of the signal with respect to the lock-in reference. Table II summarize the results of the measurements of the coupling of each mode to the calibrator, taken at a bias electric field $E_{\text {bias }}=10^{5} \mathrm{~V} / \mathrm{m}$.

Mode 3, at $2943.398 \mathrm{~Hz}$ and mode 6, at $2985.972 \mathrm{~Hz}$, are the most coupled modes to the transducer 1 . We measure an electromechanical coupling coefficient $\beta$ of about $5 \div$ $6 \times 10^{-4}$ and an effective mass which is about a factor of 3
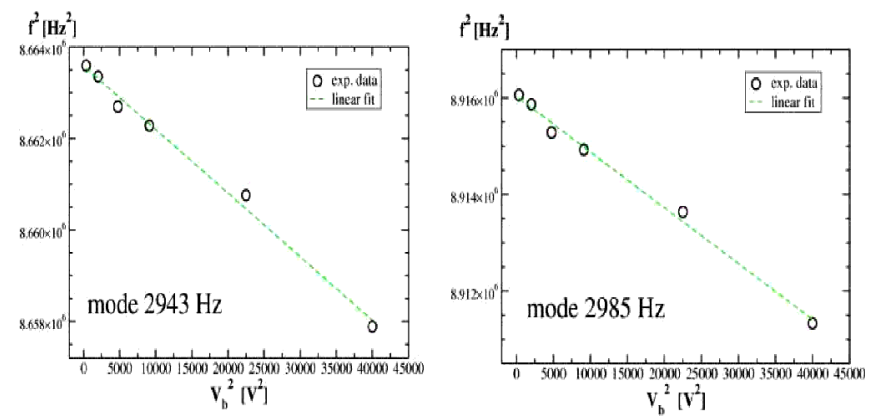

FIG. 4 (color online). Tuning curves for the two most coupled modes at $2943 \mathrm{~Hz}$ and $2985 \mathrm{~Hz}$, respectively. From the slope of the line, the mode effective mass $m_{\text {eff }}$ was estimated.
TABLE II. Tuning parameters, mechanical quality factor and time constant $\tau_{m}$ for each resonant mode measured at temperature $T=5 \mathrm{~K}$ with transducer 1 bias at an electric field $E_{\text {bias }}=$ $10^{5} \mathrm{~V} / \mathrm{m}$. The error in the frequency measurements is $\pm 0.003 \mathrm{~Hz}$. The errors in the estimation of the coupling factor are of the order of $20 \%$.

\begin{tabular}{|c|c|c|c|c|c|}
\hline Mode & $f_{o}[\mathrm{~Hz}]$ & $\beta\left[\times 10^{-4}\right]$ & $m_{\text {eff }}[\mathrm{Kg}]$ & $Q_{m}\left[\times 10^{4}\right]$ & $\tau_{m}[\mathrm{sec}]$ \\
\hline 1 & 2908.097 & 0.41 & $8.0 \pm 1.6$ & $6.8 \pm 0.1$ & $6.9 \pm 0.1$ \\
\hline 2 & 2928.143 & 1.5 & $2.3 \pm 0.4$ & $9.75 \pm 0.2$ & $10.6 \pm 0.2$ \\
\hline 3 & 2943.398 & 6.6 & $0.5 \pm 0.1$ & $5.6 \pm 0.3$ & $6.1 \pm 0.3$ \\
\hline 4 & 2949.864 & 1.4 & $2.3 \pm 0.4$ & $8.4 \pm 0.2$ & $9.1 \pm 0.2$ \\
\hline 5 & 2979.050 & 0.32 & $10.2 \pm 1.7$ & $6.3 \pm 0.2$ & $6.7 \pm 0.2$ \\
\hline 6 & 2985.972 & 5.3 & $0.60 \pm 0.1$ & $7.6 \pm 0.3$ & $8.1 \pm 0.3$ \\
\hline 7 & 3003.561 & 0.04 & $78 \pm 14$ & $9.0 \pm 0.4$ & $9.5 \pm 0.4$ \\
\hline 8 & 3023.242 & 2.2 & $1.4 \pm 0.2$ & $8.6 \pm 0.2$ & $9.1 \pm 0.2$ \\
\hline
\end{tabular}

larger than the resonating physical mass. The last columns of Table II show the quality factor $Q_{m}$ and the time constant $\tau_{m}=Q_{m} / \pi f_{m}$ for each resonant mode.

\section{B. Equivalent temperature of the coupled oscillators}

To estimate the temperature of the modes we can proceed as follows. The power spectral density at the output of the calibrator, when the backaction contribution of the room temperature amplifier is negligible, is

$$
S_{V, \mathrm{CAL}}=4 k_{B} T_{e q} \operatorname{Re}[Z(\omega)]
$$

where $\operatorname{Re}[Z(\omega)]$ was derived in Eq. (1), $T_{e q}$ is the equivalent temperature of the modes and we are considering monolateral spectra.

When an excitation is applied to the PZT at the mode resonance frequency, the voltage $V_{\text {cal }}(\omega)$ measured at the output of the FET amplifier connected to the calibrator is proportional to the voltage $V_{\mathrm{SQ}}(\omega)$ measured at the output of the two-stage SQUID of transducer 1, i.e. $V_{\text {cal }}=$ $A_{\mathrm{cal}, s q} V_{\mathrm{SQ}}(\omega)$. This is true at resonance. If we now measure the power spectrum $S_{V, \mathrm{SQ}}$ at the SQUID output, when the modes are not excited, we can evaluate the equivalent temperature of the modes using Eq. (3), where we substitute

$$
S_{V, \mathrm{CAL}}=A_{\mathrm{cal}, s q}^{2} S_{V, \mathrm{SQ}}
$$

Here we assumed that the system response is linear in the whole excitation and frequency range. Linearity has been checked for different excitation voltage. We found a linear behavior within $10 \%$.

To estimate the equivalent temperature of the modes we measured the variance $\sigma^{2}$ of the stochastic process with a spectral noise $S_{V, \mathrm{SQ}}$ at the output of the two-stage SQUID. From Eqs. (2)-(4), the variance can be written as follows 


$$
\sigma_{m}^{2}=\frac{4 k_{B} T_{e q}}{2 \pi} \frac{Q_{m}^{*}}{Q_{m}} \frac{A_{m}}{A_{\mathrm{cal}, s q}^{2}} \int_{\left(\omega_{m}-\Delta\right)}^{\left(\omega_{m}+\Delta\right)} \frac{d \omega}{\left(\omega_{m}^{2}-\omega^{2}\right)^{2}+\frac{\omega^{2} \omega_{m}^{2}}{Q_{m}^{2}}}
$$

from where we obtain the relation which links the equivalent temperature of the mode to the variance of the stochastic process with spectral noise given by $S_{V, \mathrm{SQ}}$.

We have

$$
T_{e q}=\frac{\omega_{m}^{3} A_{\mathrm{cal}, s q}^{2}}{2 k_{B} A_{m, 0} Q_{m}} \frac{Q_{m}}{Q_{m}^{*}} \sigma_{m}^{2}=\alpha_{m} \sigma_{m}^{2},
$$

where $A_{\mathrm{cal}, s q}^{2}$ comes from the calibration as described in this section, and $A_{m}$ is estimated from the Lorentzian curve fitting of each resonance of the real part of the calibrator impedance. The factor $Q_{m} / Q_{m}^{*}$ needs to be included to correct for the difference between the intrinsic quality factor $Q_{m}$ and the apparent one $Q_{m}^{*}$ measured when the two-stage SQUID is operated in flux locked loop and the cold damping is active [24]. For the measurements described here it ranges from $20 \%$ to $30 \%$ depending on the mode coupling. For the electrical resonance, not considered here in detail because uncoupled to the mechanical mode, the apparent quality factor can change by several orders of magnitude.

To estimate $\sigma_{m}$ a lock-in amplifier is used with the reference set at the resonance frequency of the mode. The lock-in amplifier output magnitude $r$ and angular phase $\phi$ are then sampled at regular time intervals. The amplitude decay time constant of the lock-in amplifier is chosen equal to the sampling time $\tau_{s}=\tau_{l k}$. To observe the free evolution of the $m_{t} h$ mode the lock-in amplifier time constant is chosen smaller than the time constant of the mode, $\tau_{l k}<\tau_{m}$, but large enough that the lock-in amplifier works as a bandpass filter and makes the contribution of the broadband noise of the SQUID and the tails of the neighboring modes negligible. The mean square amplitude $\left\langle r^{2}\right\rangle$ of the lock-in amplifier input signal magnitude, equal to the variance of the total narrow-band noise $V_{n b}^{2}$, is given by [31]

$$
\left\langle r^{2}\right\rangle=V_{n b}^{2}=\left(1+\frac{\tau_{l k}}{\tau_{m}}\right)\left(\sigma_{0}^{2}-\frac{S_{w b}}{2 \tau_{l k}}\right),
$$

where $\sigma_{0}^{2}$ is the variance of the power spectral density output, and $S_{\mathrm{wb}}$ is the power spectral density of the SQUID wideband noise. Generally the factor $\tau_{m} /\left(\tau_{m}+\right.$ $\left.\tau_{l k}\right) \sim 1$ in our case.

The stochastic process $r^{2}$ is the sum of two independent Gaussian processes, the in-phase and quadrature lock-in amplifier output. If the signal is absent or in general if its average contribution is negligible with respect to the noise, the variable $r^{2}$ will have the exponential distribution $F\left(r^{2}\right)=\frac{1}{2 \sigma_{m}} e^{-\left(r^{2} / 2 \sigma_{m}\right)}$.

The estimate of $\sigma_{m}^{2}$, is then performed by sampling the magnitude $r$ at regular time intervals, with sampling time $\tau_{s} \ll \tau_{m}$. A subset of data is created by extracting a data point every resonator time constant $\tau_{m}$ in order to get uncorrelated samples. After a large number of samples is collected, a histogram $N\left(r^{2}\right)$ is built, where $N$ is the number of samples in a given bin around $r^{2}$. The histogram is fit with the exponential distribution described above and the mean square amplitude $\left\langle r^{2}\right\rangle$ is then extracted as fitting parameter.

In the absence of excess or amplifier backaction noise, the quantity $\left\langle r^{2}\right\rangle$ is proportional to the thermal vibrational energy in the antenna mode. The constant of proportionality $\alpha_{m}$ was used to rescale the recorded values of $\left\langle r^{2}\right\rangle$ to antenna energy. For the two most coupled modes at frequencies 2943 and $2985 \mathrm{~Hz}$ we found the calibration factor $\alpha_{m}$ to be $\alpha_{2943}=(7.0 \pm 1.5) \times 10^{8}\left[\mathrm{~K} / \mathrm{V}^{2}\right]$ and $\alpha_{2985}=$ $(1.1 \pm 0.2) \times 10^{9}\left[\mathrm{~K} / \mathrm{V}^{2}\right]$ respectively.

Graphs (a) and (b) in Fig. 5 show the energy distribution estimated for the modes at frequencies 2943 and $2985 \mathrm{~Hz}$ during three hours of acquisition. The equivalent temperature for both modes is obtained by fitting the exponential distribution.

The slope of the distribution corresponds to a temperature of $(7.0 \pm 2) \mathrm{K}$ for the mode at $2943 \mathrm{~Hz}$ and $(9 \pm 2) \mathrm{K}$ for the mode at $2985 \mathrm{~Hz}$, the error arising mainly from the calibration uncertainty. The equivalent temperatures are consistent, within two sigma, with the thermodynamic temperature of the sphere. No significant difference is observed in the equivalent temperature of the mode between night and day acquisitions.

\section{Force calibration and strain sensitivity}

While for a bar detector it is relatively straightforward to relate the strain produced by a calibrator located on one of the bar faces with the strain from a gravitational wave signal, the same cannot be said for a spherical detector. In a spherical detector a calibration hammer stroke excites all five spheroidal modes, with relative amplitudes depending on the position of the stroke on the sphere surface. When only one excitation signal is used and not all the six transducers on the TI configuration are read out simultaneously, like for the MiniGRAIL test run described in this paper, we can only calibrate the detector for a particular set of forces $\mathbf{F}_{\mathbf{m}}$ applied to each spheroidal mode [9]. Such a combination of forces does not necessarily represent a $\mathrm{GW}$ excitation.

In the experiment described here, the calibration signal is generated by a piezoelectic ceramic located at position $\left(\theta=18^{\circ}, \phi=135^{\circ}\right)$. By using Eq. 45 in [9], derived previously in $[3,5,26]$, one finds that a piezo in such a location excites a combination of the 5 spheroidal modes given by the vector $(0,0.13,-0.49,0.5,-1)$, normalized to the maximum value of its elements. Such a combination is equal, within $20 \%$ tolerance, to the one generated by a circularly polarized gravitational wave coming from direction $\left(\theta=20^{\circ}, \phi=135^{\circ}\right)$. According to [9] such a wave 

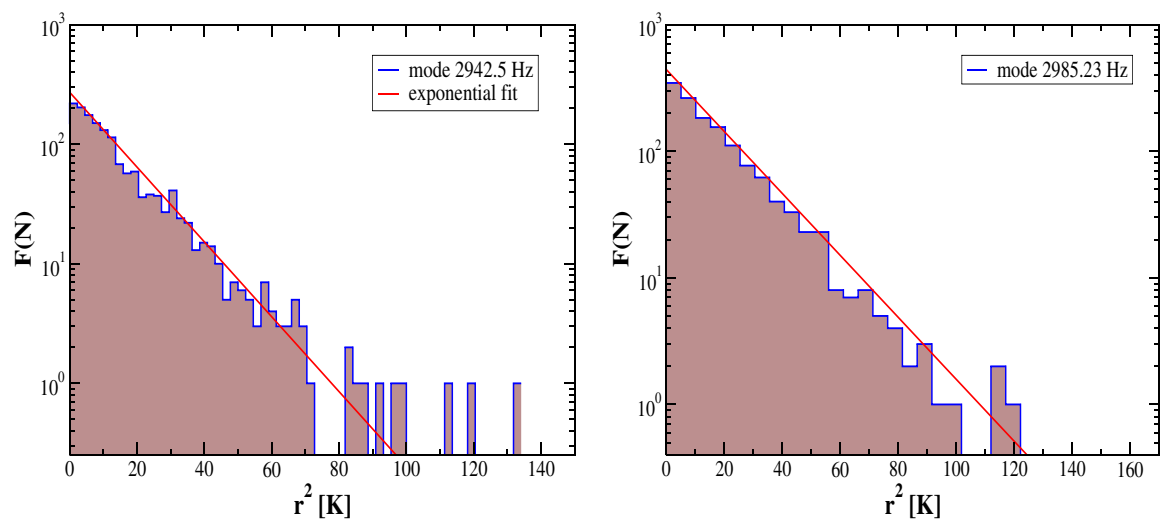

FIG. 5 (color online). Exponential distributions of the mean square amplitude $\left\langle r^{2}\right\rangle$ for the modes at 2943 and $2985 \mathrm{~Hz}$. The variance $\sigma_{m}^{2}$ obtained from the fit of the exponential distribution gives an equivalent temperature of the two modes of $(7.0 \pm 2)$ and $(9 \pm 2) \mathrm{K}$ respectively.

direction is almost optimal for the transducer configuration described in this paper.

To calibrate the detector in force we proceed as follows. Using the calibration piezo described above we excite at resonance each detector mode and record the SQUID output. When the detector $m_{t} h$ mode is excited at resonance $\omega_{m}$ with an energy $\epsilon=\frac{1}{2} k_{B} T_{m}$, the power spectral density measured at the SQUID output is

$$
S_{V, \mathrm{SQ}}=\frac{4 k_{B} T_{m} \operatorname{Re}\left[Z\left(\omega_{m}\right)\right]}{A_{\mathrm{cal}, s q}^{2}}\left[\frac{V^{2}}{H z}\right]
$$

where $\operatorname{Re}[Z(\omega)]$ is the real part of the calibrator impedance and was derived in Eq. (2). The force power spectral density of the detector mode excited at a temperature $T_{m}$, when backaction is negligible as in our case, is given by

$$
S_{F, m}=\frac{4 k_{B} T_{m} m_{\mathrm{eff}, m} \omega_{m}}{Q_{m}}\left[\frac{N^{2}}{H z}\right]
$$

where $m_{\mathrm{eff}, m}$ is the effective mass of the mode $m$, which can be estimated from the tuning curves of the sensor transducer if the bias voltage and the gap are known, see Table II.

From the ratio of Eqs. (8) and (9) we get

$$
\frac{S_{v, s q}}{S_{F, m}}=\frac{Q_{m} \operatorname{Re}\left[Z\left(\omega_{m}\right)\right]}{m_{\mathrm{eff}, m} \omega_{m} A_{\mathrm{cal}, s q}^{2}}\left[\frac{V^{2}}{N^{2}}\right] .
$$

At each mode resonance Eq. (10) becomes

$$
\left(\frac{S_{V, \mathrm{SQ}}}{S_{F, m}}\right)_{\omega=\omega_{m}}=\frac{A_{m, c}}{m_{e f f, m} A_{\mathrm{cal}, s q}^{2}} \frac{Q_{m, c}^{3}}{\omega_{m}^{5}}\left[\frac{V^{2}}{N^{2}}\right] .
$$

We observed small changes in the frequencies and quality factors of the resonant modes when the calibrator bias was set on and off. This is due to the fact that the calibrator resonance is tuned to the spheroidal modes as well. This effect should be included while deriving Eq. (11). This could be done by using the different measured values for $\omega_{m}$ and $Q_{m}$, which appear in Eq. (10) and $\operatorname{Re}\left[Z\left(\omega_{m}\right)\right]$, respectively. For the sake of simplicity, we neglected these corrections considering that they amount to less than 5\% and are therefore smaller than the total uncertainties in the calibration procedure.

The relations derived so far are valid at resonance. As a first approximation one can define the transfer function $G_{\mathrm{SQ}, F}$ for gravitational wave signals, which converts a combination of quadrupolar forces into SQUID output voltage, as a product of poles and zeros where the poles are derived from the polynomial fit of the SQUID noise spectrum and the zeros are chosen to fit the measured amplitudes at resonance given by Eq. (11). The transfer function for the applied calibration signal becomes then

$$
G_{\mathrm{SQ}, F}(\omega)=H_{m, \mathrm{cal}}(\omega) \frac{\prod_{k=1}^{N_{r}}\left(j \omega-r_{k, m}\right)\left(j \omega-r_{k, m}^{*}\right)}{\prod_{k=1}^{N_{p}}\left(j \omega-p_{k}\right)\left(j \omega-p_{k}^{*}\right)} .
$$

In the equation above $N_{p}>N_{r}$ and $H_{m \text {,cal }}(\omega)$ is a force calibration constant which can be experimentally determined from the calibration measurement at resonance. All the terms included in Eqs. (11) and (12) are experimentally derived from the calibration, from the tuning curves and from direct measurement of the modes quality factor. The transfer function is experimentally measured at resonance and only approximated out of resonance.

Once a parametrization of the transfer function is available, Eq. (12), one can derive the detector strain sensitivity using Eq. 26 of [9]. We use $m_{s}=1300 \mathrm{Kg}$ for the sphere physical mass, $R=34 \mathrm{~cm}$ for the radius and $\chi=0.325$, which is valid for the $\mathrm{CuAl}$ alloy. The MiniGRAIL strain sensitivity with three transducers coupled to the spheroidal modes, but only one used for the readout, is shown in Fig. 6. The readout transducer was biased with a constant electric field of $E=10^{7} \mathrm{~V} / \mathrm{m}$. As discussed above, the experimental strain curve gives and estimation of the detector sensitivity only for a particular combination of sphe- 


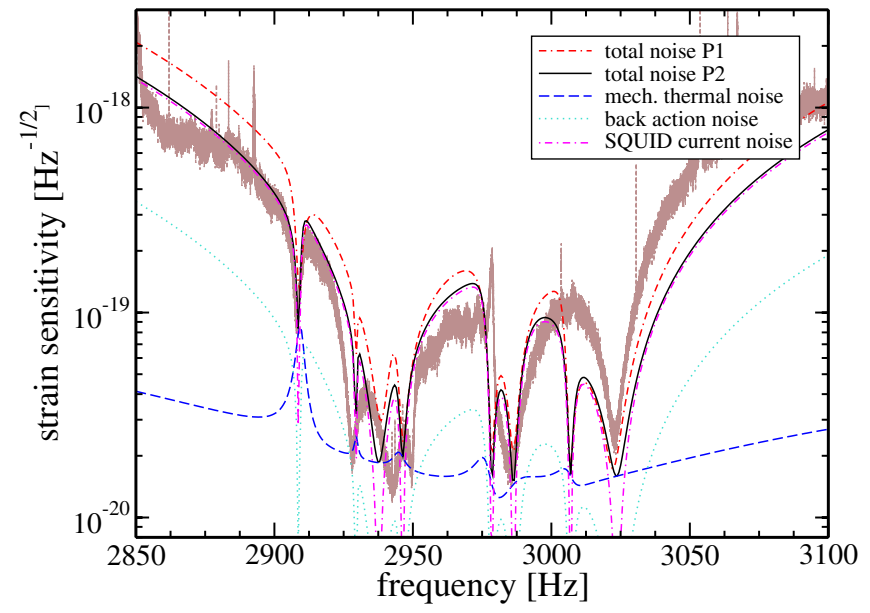

FIG. 6 (color online). MiniGRAIL strain sensitivity at a thermodynamic temperature $T=5.2 \mathrm{~K}$ with three transducers placed on the sphere, but single transducer readout. The sensitivity has been estimated for a particular combination of the 5 spheroidal modes as derived by exciting the mode using a piezoelectric transducer (PZT) located at position $\mathrm{P} 1 \quad(\theta=$ $18^{\circ}, \phi=135^{\circ}$ ). The dot-dash-dashed curve (total noise Pl) shows the strain sensitivity calculated for a simulated hammerstroke excitation from the PZT location P1 using the electromechanical model described in [9]. For the simulation we used the detector parameters discussed here in the text. A better matching between the experimental data and the simulation is obtained when the simulated hammer stroke is given at the position P2 $\left(\theta=27^{\circ}, \phi=135^{\circ}\right)$ (continuous line curve labeled total noise $P 2)$. The other curves shows the contribution to the stray sensitivity of the thermal noise (dashed line), backaction noise (dotted line) and SQUID additive current noise (dot-dashed line).

roidal modes corresponding to a gravitational wave coming from the $\left(\theta=20^{\circ}, \phi=135^{\circ}\right)$ direction.

We obtained a peak strain sensitivity of $(1.5 \pm 0.6) \times$ $10^{-20} \mathrm{~Hz}^{-1 / 2}$ at $2942.9 \mathrm{~Hz}$ and a strain sensitivity of about $5 \times 10^{-20} \mathrm{~Hz}^{-1 / 2}$ over a bandwidth of $30 \mathrm{~Hz}$. This corresponds to a strain amplitude of $h \simeq 2.5 \times 10^{-18}$ at $3 \mathrm{kHz}$ for a burst signal of $1 \mathrm{~ms}$ [32]. For a sphere of $68 \mathrm{~cm}$ in diameter like the one of MiniGRAIL, it is equivalent to a displacement sensitivity, at $3 \mathrm{kHz}$, of $1.6 \times 10^{-19} \mathrm{~m}$. When optimal filtering is applied to the output signal, the detector is sensitive to burst signals with an impulse energy of about $T_{\text {pulse }} \sim 50 \mathrm{mK}$ as can be derived calculating the noise temperature using the experimental data. The calculated sensitivity curves in Fig. 6, are obtained using the model described in [9]. The dot-dash-dashed curve shows the strain sensitivity calculated for a simulated hammerstroke excitation applied to the same point $\mathrm{P} 1$ on the sphere where the PZT is placed, i.e. $\left(\theta=18^{\circ}, \phi=135^{\circ}\right)$. The simulated signal describes reasonably well the strain sensitivity.

The best fitting strain sensitivity has been obtained for a simulated hammer-stroke applied at the sphere surface point $\mathrm{P} 2\left(\theta=27^{\circ}, \phi=135^{\circ}\right)$. The result is shown with the continuous line. In this case the third mode is more excited. The agreement with the experimental data is remarkable, considering the amount of fitting parameters involved in the simulation. The difference of about $7^{\circ}$ in the angle $\theta$ of the experimental and simulated excitation position could be explained considering the fact that a spherical detector with only three transducers in the position 1, 2 and 5 like the one considered here is far from being symmetric. In [27] as well the authors had to perform a rotation of the reference frame of the spherical harmonics that determined the pattern matrix to be able to explain their experimental results. In their case the transducers were not as massive as here and, above all, they used six transducers positioned in the symmetric TI configuration. The resulting mixing of the spheroidal modes could explain the discrepancy between the measured and the simulated sensitivity curves. In order to address more accurately this issue one should place on the sphere at least 5 calibrators to measure the detector response to each of the 5 spheroidal modes.

The contribution to the strain sensitivity of the well known noise sources are plotted in Fig. 6 as well. At resonance, the sensitivity is limited by mechanical thermal noise of the transducer mass. Out of resonance the sensitivity is limited by the SQUID additive current noise. The backaction noise of the SQUID is about an order of magnitude smaller. The electrical thermal noise of the superconducting transformer, not shown in the graph, is negligible because the electrical mode is well decoupled from the mechanical ones due to its higher resonant frequency.

From the measurement of the variance of the most coupled modes and the simulated data we can conclude that, within the experimental accuracy, the MiniGRAIL peak sensitivity is currently limited by the thermal noise generated by the transducer mass. Some of the modes, however, show excess noise generated probably from electromagnetic interferences or spurious mechanical coupling. A better estimate of the transfer function of each spheroidal mode is necessary in order to properly characterize the detector. As described in detail in [9] when $N<$ 5 transducers are used to read out the sphere one needs to generate calibration forces from a set of 5 or more calibrators located at different positions on the sphere surfaces.

To operate the sphere as a omnidirectional GW detector the six transducers in the TI configuration have to be read simultaneously. In this case, as shown in [9], only one force calibrator, mounted at an arbitrary position on the sphere surface. This is due to the existing one-to-one relation between the mode channels and the forces acting on the spheroidal modes. When a full transducer TI configuration is used the calibration is easier and it can be further simplified by using nonresonant force calibrators and implementing a calibration coil in each readout chain to 


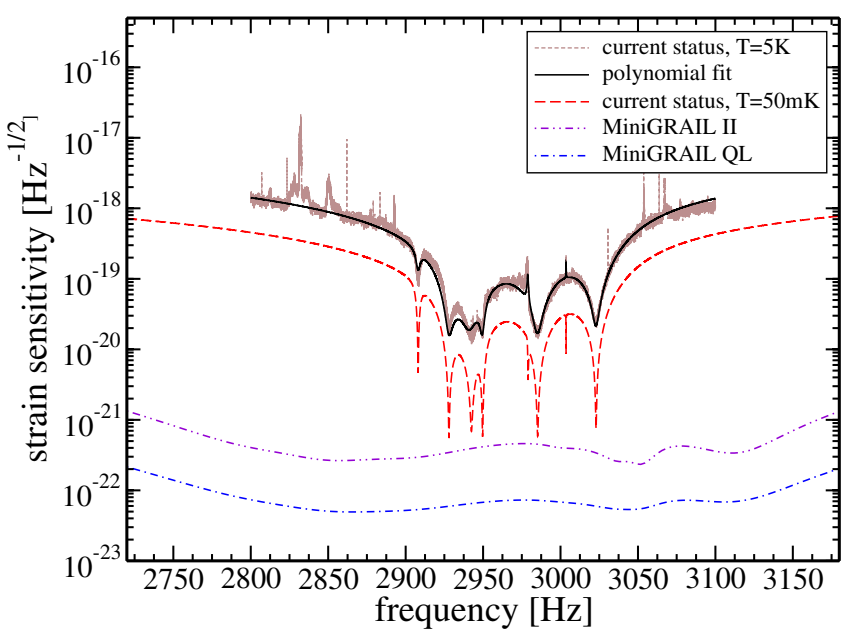

FIG. 7 (color online). The measured strain sensitivity of MiniGRAIL is shown together with the predicted sensitivity for future detector configurations. The continuous line is a polynomial fit of the measured strain sensitivity. The dashed line shows the expected sensitivity for the detector operating at $T=50 \mathrm{mK}$ with the same three transducers configuration presented in this paper. The dot-dot-dashed line (MiniGRAIL II) shows the sensitivity achievable with available technology, namely $T / Q \sim 2.5 \times 10^{-8} \mathrm{~K}$ and SQUID energy resolution $E=70 \hbar$. The dot-dashed curve gives the sensitivity for a quantum limited detector (MiniGRAIL $Q L$ ) with $T / Q \sim 1 \times 10^{-9} \mathrm{~K}$.

directly measure the transducer impedance. The data reduction and filtering techniques used for bar detector can be applied to a spherical detector as well [9], however special filters and detection strategies need to be developed to fully retrieve the information about the wave arrival time, direction and polarization [33].

The measurements presented here have to be considered as a first test bed for the following engineering and science runs of MiniGRAIL. The sensitivity is expected to improve of at least 1 order of magnitude when the detector will operate at a thermodynamical temperature below $50 \mathrm{mK}$.

In Fig. 7 the measured strain sensitivity is shown together with predicted sensitivity for possible future detector configurations. A polynomial fit of the strain sensitivity is shown as well. The poles and zeros obtained from the fit can be used to build the matched filters for GW detection
[9]. The expected strain sensitivity of MiniGRAIL is shown for the detector operating at $T=50 \mathrm{mK}$ with the same three transducers configuration presented here.

The figure shows the expected sensitivity for MiniGRAIL II. In this configuration MiniGRAIL operates with 6 capacitive transducers placed in the TI configuration where the electrical modes are coupled to the mechanical ones. We consider $T / Q=2.5 \times 10^{-8} \mathrm{~K}$ and a SQUID coupled energy resolution $E_{\text {coupled }}=70 \hbar$. Those values are achievable within the current technology [9]. The sensitivity of MiniGRAIL operating at the quantum limit is also shown. MiniGRAIL can reach a peak sensitivity of about $6 \times 10^{-23} \mathrm{~Hz}^{-1 / 2}$ and a bandwidth larger than $400 \mathrm{~Hz}$ at a sensitivity of $1 \times 10^{-22} \mathrm{~Hz}^{-1 / 2}$.

\section{CONCLUSIONS}

We have operated at a thermodynamic temperature $T=$ $5.2 \mathrm{~K}$ a spherical resonant detector equipped with a capacitive resonant transducers coupled to a two-stage SQUID amplifier. Our two-stage SQUID amplifier is one of the most sensitive amplifiers employed so far on a GWs resonant detector. We measured an additive coupled energy resolution of $(700 \pm 100) \hbar$. We achieved a peak strain sensitivity of $1.5 \times 10^{-20} \mathrm{~Hz}^{-1 / 2}$ at $2942.9 \mathrm{~Hz}$. A strain sensitivity of better than $5 \times 10^{-20} \mathrm{~Hz}^{-1 / 2}$ has been obtained over a bandwidth of $30 \mathrm{~Hz}$. We expect an improvement of more than 1 order of magnitude when the detector will operate at $50 \mathrm{mK}$. This result should be considered as the first step towards the realization of an ultracryogenic spherical gravitational wave detector.

\section{ACKNOWLEDGMENTS}

The authors would like to acknowledge precious discussions with Jean-Pierre Zendri, Paolo Falferi, Andrea Vinante, G. V. Pallottino, and Alberto Lobo. We are grateful to Hibbe van der Mark for his technical help. This work has been partially financially supported by Integrated Large Infrastructures for Astroparticle Science (ILIAS) of the Sixth Framework Programme of the European Community.
[1] E. Coccia, J. A. Lobo, and J. A. Ortega, Phys. Rev. D 52, 3735 (1995).

[2] J. A. Lobo, Phys. Rev. D 52, 591 (1995).

[3] S. M. Merkowitz, Phys. Rev. D 58, 062002 (1998).

[4] C.Z. Zhou and P.F. Michelson, Phys. Rev. D 51, 2517 (1995).

[5] T. R. Stevenson, Phys. Rev. D 56, 564 (1997).
[6] A. de Waard et al., Classical Quantum Gravity 23, S79 (2006).

[7] O.D. Aguiar et al., Classical Quantum Gravity 23, S239 (2006).

[8] C. D. Tesche and J. Clarke, J. Low Temp. Phys. 29, 301 (1977).

[9] L. Gottardi, Phys. Rev. D 75, 022002 (2007). 
[10] A. de Waard et al., Classical Quantum Gravity 21, S465 (2004).

[11] P. Falferi, M. Bonaldi, M. Cerdonio, A. Vinante, R. Mezzena, G. A. Prodi, and S. Vitale, Appl. Phys. Lett. 88, 062505 (2006).

[12] M. Podt, L. Gottardi, A. de Waard, G. Frossati, and J. Flokstra, Supercond. Sci. Technol. 16, 1531 (2003).

[13] A. Vinante, M. Bonaldi, P. Falferi, M. Cerdonio, R. Mezzena, G. A. Prodi, and S. Vitale, in Proceedings of the "SQUID 2001 Conference" 2001, Stenungsbaden, Sweden [Physica C (Amsterdam) 368, 176 (2002)].

[14] A. de Waard et al., Classical Quantum Gravity 22, S215 (2005).

[15] Quantum Design, 11578 Sorrento Valley Road, San Diego, CA.

[16] M. Podt, M. J. van Duuren, A. W. Hamster, J. Flokstra, and H. Rogalla, Appl. Phys. Lett. 75, 2316 (1999).

[17] M. J. van Duuren, G. C. S. Brons, J. D. J. Adelerhof, and H. Rogalla, J. Appl. Phys. 82, 3598 (1997).

[18] L. Gottardi, Ph.D. thesis, Leiden University, Leiden, The Netherlands, 2004, http://www.sron.nl/ lucianog/ Gottardi_PhD_2004.pdf.

[19] R. Mezzena, A. Vinante, P. Falferi, S. Vitale, M. Bonaldi, G. A. Prodi, M. Cerdonio, and M. B. Simmonds, Rev. Sci. Instrum. 72, 3694 (2001).

[20] G. M. Harry, J. Houser, and K. A. Strain, Phys. Rev. D 65,
082001 (2002).

[21] P. Carelli, M. G. Castellano, G. Torrioli, and R. Leoni, Appl. Phys. Lett. 72, 115 (1998).

[22] L. Gottardi et al., Classical Quantum Gravity 21, S1191 (2004).

[23] T. R. Stevenson and H. J. Haucke, in Proceedings of the 1st Edoardo Amaldi Conference on Gravitational Waves (World Scientific, Singapore, 1995), p. 390.

[24] L. Baggio et al., Phys. Rev. Lett. 94, 241101 (2005).

[25] M. Bassan, Ph.D. thesis, Stanford University, Stanford, CA, 1985.

[26] J. A. Lobo, Mon. Not. R. Astron. Soc. 316, 173 (2000).

[27] S. M. Merkowitz and W. W. Johnson, Phys. Rev. D 56, 7513 (1997).

[28] H. J. Paik, J. Appl. Phys. 47, 1168 (1976).

[29] J. P. Richard, Phys. Rev. Lett. 52, 165 (1984).

[30] Y. Ogawa and P. Rapagnani, Nuovo Cimento Soc. Ital. Fis. 7C, 21 (1984).

[31] P. Bonifazi, V. Ferrari, S. Frasca, G. V. Pallottino, and G. Pizzella, Nuovo Cimento Soc. Ital. Fis. 1C-6, 465 (1978).

[32] E. Coccia, in Proceedings of the International Conference on Gravitational Waves: Sources and Detectors (World Scientific, Singapore, 1996), p. 201.

[33] M. A. Gasparini and F. Dubath, Phys. Rev. D 74, 122003 (2006). 